\title{
Les hommes et leurs volcans : représentations et gestion des phénomènes volcaniques en Polynésie (Hawaii et Royaume de Tonga)
}

\section{Cécile Quesada}

\author{
CpenEdition \\ Journals \\ Édition électronique \\ URL : http://journals.openedition.org/jso/382 \\ DOI : $10.4000 /$ jso.382 \\ ISSN : $1760-7256$ \\ Éditeur \\ Société des océanistes \\ Édition imprimée \\ Date de publication : 1 décembre 2005 \\ Pagination : 64-73 \\ ISSN : 0300-953x
}

\section{Référence électronique}

Cécile Quesada, «Les hommes et leurs volcans : représentations et gestion des phénomènes volcaniques en Polynésie (Hawaii et Royaume de Tonga) », Journal de la Société des Océanistes [En ligne], 120-121 | Année 2005, mis en ligne le 27 novembre 2008, consulté le 03 mai 2019. URL : http:// journals.openedition.org/jso/382 ; DOI : 10.4000/jso.382 


\section{Les hommes et leurs volcans : représentations et gestion des phénomènes volcaniques en Polynésie (Hawaii et Royaume de Tonga)}

par

Cécile QUESADA*

\section{RÉSUMÉ}

Cet article a pour objet l'étude des relations que deux sociétés de Polynésie ont tissées avec les volcans. Dans le Hawaii pré-chrétien, les hommes avaient anthropomorphisé les phénomènes volcaniques et fait des divinités chtoniennes, qui les personnifiaient, l'un des termes de relations généalogiques qui conditionnaient l'efficacité de rites propitiatoires. Ces derniers visaient à prévenir ou à stopper les éruptions qui étaient conçues comme déclenchées par ces déités suite au non-respect, par les hommes, des règles de la vie en société. À Niuafo'ou (Tonga), les éruptions sont interprétées aujourd'hui comme des signes de la colère du Dieu chrétien, provoquée par des transgressions morales ou sociales. Le volcan est alors conçu comme un instrument divin, visant à châtier les actes qui mettent en danger l'ordre social. D'une société à l'autre, d'une époque à l'autre, on peut ainsi faire état d'une certaine continuité dans le lien que les hommes construisent avec les phénomènes volcaniques.

Mots-CLÉS: Polynésie, Hawaii, Tonga, systèmes de représentations, rites propitiatoires, relations avec les phénomènes volcaniques.

De nombreuses communautés d'Océanie vivent à proximité de volcans et sont souvent confrontées à des aléas géophysiques comme les éruptions, les séismes ou les tsunami. Une

\begin{abstract}
This article focuses on the study of the relations that two polynesian societies have woven with the volcanoes. In the pre-Christian Hawaii, men had anthropomorphised these volcanic phenomena, and considered the chtonian divinities that personified them as one of the parts of genealogical relationships that conditioned the efficiency of propitiatory rites. These rites were meant to prevent or to stop eruptions, conceived as triggered off by these deities after men had transgressed the rules of life in the society. In Niuafo'ou (Tonga) today, the eruptions are interpreted as signs of the Christian god's wrath after moral or social transgressions. The volcano is thus considered as God's tool, aiming at punishing the acts that threaten the social order. Thus, from one society to the other, from one period to the other, we can observe a certain continuity in the link men build with the volcanic phenomena.
\end{abstract}

Keywords: Polynesia, Hawaii, Tonga, systems of representations, propitiatory rites, relations with volcanic phenomena.

anthropologie des relations que ces sociétés entretiennent avec leur environnement ne peut donc pas faire l'économie d'une analyse des rapports qu'elles ont pu tisser avec ces

* Doctorante en anthropologie, CREDO-Marseille, cequesada@wanadoo.fr 
phénomènes d'origine tellurique, même si ceux-ci sont généralement conçus dans nos sociétés comme des composants du milieu non maîtrisables.

$\mathrm{Au}$ sein des sociétés polynésiennes exposées à des phénomènes volcaniques, j'ai consacré une étude au cas de Hawaii ${ }^{1}$, puis j'ai effectué une enquête de terrain dans le Royaume de Tonga ${ }^{2}$ afin d'observer directement la manière dont ces hommes se pensent en relation avec leur volcan et interagissent avec lui. J'ai pu ainsi remarquer que, malgré les différences qui existent entre les deux cultures et les deux périodes étudiées, on peut certes mettre au jour des différences concernant les rapports tissés avec les phénomènes volcaniques, mais aussi et surtout, on retrouve une continuité notable des représentations et des pratiques. L'objectif de cet article est donc d'explorer cette continuité, à travers l'analyse des systèmes de conceptions, de savoirs et de savoir-faire que ces deux sociétés ont élaborés à l'endroit des volcans et de leurs activités associées ${ }^{3}$.

La première partie de cet article porte sur un ensemble de représentations mythiques et de pratiques rituelles qui ont été observées à Hawaii à l'époque du contact européen et reportées dans des textes écrits par des explorateurs, des missionnaires chrétiens, des natifs, des chercheurs et des visiteurs. La deuxième partie s'intéresse à la manière dont les habitants de l'île de Niuafo'ou (Tonga) conçoivent, à l'heure actuelle, leurs relations avec leur île volcanique et les phénomènes afférents. Une troisième partie consiste en une comparaison de ces deux systèmes de représentations, destinée à montrer ce qui, au-delà des différents contextes historiques et religieux, les rapproche. Il s'agit ainsi de comprendre la logique sous-jacente qui a permis - et permet encore - à ces deux sociétés océaniennes d'interpréter les phénomènes volcaniques et de les « saisir » pour en contrôler - dans une certaine mesure - l'occurrence et l'impact.

\section{Les représentations hawaiiennes des phénomènes volcaniques}

L'archipel d'Hawaii (Fig. 1) est constitué d'une chaîne d'îles volcaniques, parmi lesquelles seule l'île la plus méridionale, Hawai'i, est encore active. Les éruptions effusives peuvent y détruire des terres, des villages, et même parfois des vies humaines. Depuis la découverte de cette société par les Occidentaux, de riches corpus de mythes, de traditions orales et de pratiques cérémonielles ont été compilés. Les cosmogonies narrent la création du monde, qui a consisté en un processus d'engendrements successifs issus de l'imprégnation de principes femelles par des principes mâles (Handy, 1927 : 22). Ces engendrements, donnant naissance à des formes animales et végétales de plus en plus complexes, ont eu lieu durant le stade originel du po, "l'obscurité ». C'est au moment de la naissance des hommes et des dieux que l'on est passé au stade du $a o$, la « lumière du jour », qui correspond au monde visible tel que les Hawaiiens le connaissaient (Valeri, 1985: 4). Dans ces conceptions, un océan recouvrait tout, préexistant à la formation du monde. Les terres ont alors été remontées à la surface lors d'une pêche divine. Sa description varie selon les récits de tradition orale, mais elle met généralement en scène le dieu et héros civilisateur Maui.

Le feu volcanique, ahi pele ${ }^{4}$, est quant à lui originaire du monde souterrain. Il y est gardé et entretenu par des divinités liées généalogiquement à Pele, la déesse des volcans, qui en est l'incarnation (Dunis, 1990: 305 ; Kamakau, 1976 : 117). C'est ce feu souterrain qui surgit à la surface de la terre lors des éruptions volcaniques. Un cycle complexe de chants narrant l'histoire de Pele était enseigné aux pratiquants de la hula, un ensemble de chants et de danses sacrés dont les patronnes étaient Pele et sa sœur Hiiaka (Linge, 1975 : 70 ; Pukui et al., 1993 : xii). Ce cycle mythique raconte que Pele est originaire d'une autre île (souvent située dans l'archipel des Îles de la Société) d'où elle s'est enfuie pour chercher une demeure. Après de longues errances, elle s'est installée dans le cratère du Kilauea (île d'Hawai'i), là où le feu brûlait dans les entrailles de la terre. Depuis, elle déclenche éruptions et séismes lorsqu'elle se met en colère, irritée par le non-respect par les hommes de certaines règles de la vie en société, la transgression de tabous, ou les démonstrations d'arrogance ou d'avarice (Westervelt, 1963).

Les activités volcaniques sont considérées comme les manifestations physiques de Pele ou

1. Cette société, ainsi que celle des Maori de Nouvelle-Zélande et de Tonga, toutes les trois prises à l'époque du contact européen, ont fait l'objet de mon mémoire de DEA (Quesada, 2000).

2. Cette recherche de terrain portant sur la société tongienne contemporaine a duré vingt-deux mois, de 2001 à 2003 (thèse de doctorat en cours de préparation).

3. Malgré une différence d'échelle (Hawai'i étant bien plus vaste que Niuafo'ou), les éruptions qui frappent ces deux sociétés sont de même type (effusif, c'est-à-dire provoquant des coulées de lave) et ont donc des impacts similaires sur l'environnement et sur les hommes et leurs biens.

4. Littéralement, ahi signifie feu, et pele désigne tout ce qui est lié au volcanisme. 
Niihau $0>^{\text {Kauai }}$

ARCHIPEL DE HAWAII
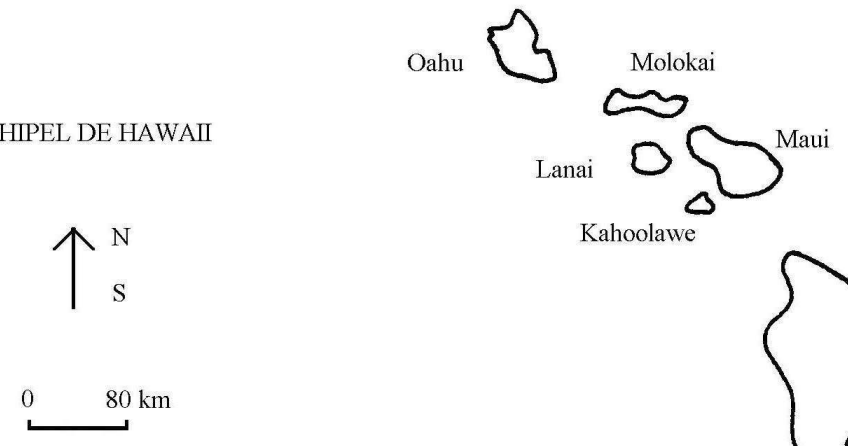

Kahoolawe

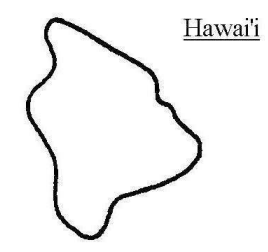

- Frontière de district

Volcan endormi

A Volcan actif

Champs de lave

$\uparrow_{\mathrm{S}}^{\mathrm{N}}$
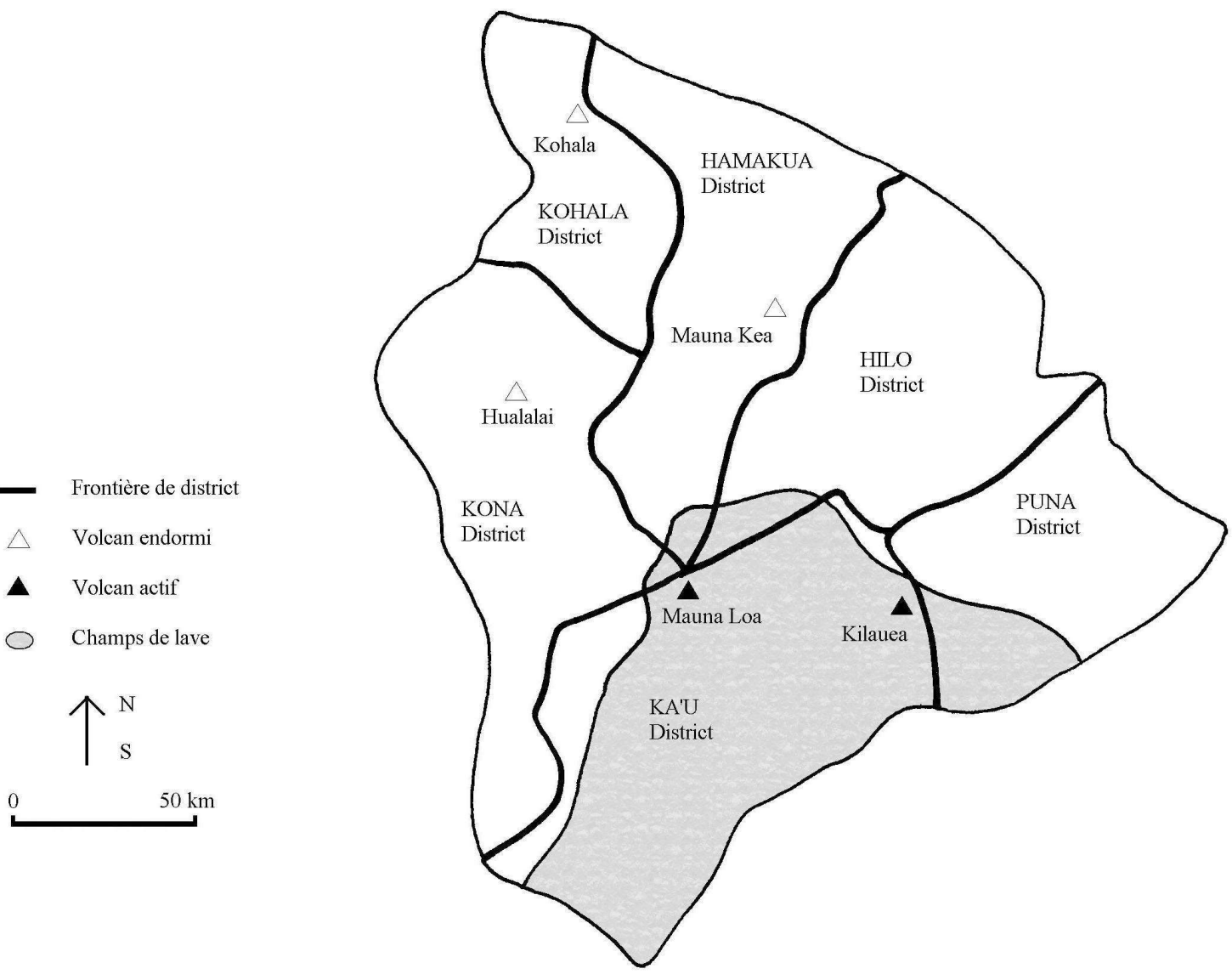

FIG. 1. - Localisation de l'île d'Hawai'i (et du district de Kan) dans l'archipel d'Hawaii. 
de ses proches parents, les « esprits du feu ». En effet, dans la religion hawaiienne, chaque phénomène naturel est appréhendé sur la base du principe des kino lau ou « corps multiples des divinités » (Salhins, 1995: 166). Ce principe est le suivant :

« Each deity is defined by its manifestations, which reveal his attributes, or predicates. As elsewhere in Polynesia [...], these manifestations can take three main forms : natural phenomena, particularly biological species ; living human forms ; and artificial forms, or anthropomorphic images. » (Valeri, $1985: 9)$

Les phénomènes telluriques, à l'image des autres composants de l'environnement, sont donc anthropomorphisés : ils sont les manifestations, les «corps multiples» de Pele et des membres de sa famille de divinités chtoniennes. En outre, les personnalités de ces déités semblent correspondre à la perception qu'ont les hommes de l'impact des éruptions et des séismes qu'elles personnifient. Ainsi, Pele, déesse impulsive, impétueuse, colérique et rancunière, incarne des phénomènes volcaniques violents, destructeurs, voire meurtriers.

Comme il a été suggéré plus haut, les divinités qui personnifient les différents phénomènes de nature ou d'origine volcanique sont liées entre elles par des rapports de parenté. Ainsi, ce sont les frères et sœurs de Pele qui incarnent les orages ou les fumées qui apparaissent au cours des éruptions, tandis que son oncle paternel, Lono-Makua, est associé à la fertilité des terres volcaniques (Beckwith, 1970 : 167). Les séismes sont aussi des manifestations de la présence de Pele ou des membres de sa famille dans un district. Il paraît possible d'en déduire que le lien généalogique que les Hawaiiens établissent entre les différents phénomènes telluriques constitue une représentation symbolique de leur lien géologique et en traduisent la connaissance. Les systèmes de représentations, au-delà des croyances, sont donc aussi les produits de la connaissance empirique que les hommes ont de leur environnement, née d'une longue cohabitation avec les volcans et leurs activités.

Afin de gérer l'occurrence des éruptions et de la sismicité associée, les Hawaiiens pratiquaient deux types de rites : des sacrifices propitiatoires et des rites funéraires.

\section{Les sacrifices propitiatoires}

Dans la religion hawaiienne, "propitiatory rites are meant to avert potential disorder or favor the realization of a project or a desired state » (Valeri, $1985: 41$ ). Les offrandes avaient lieu, soit dans des temples (heiau) que les hommes érigeaient et dédiaient à la déesse Pele, soit près des cratères qui étaient des domaines sacrés créés par cette divinité. Les dons - enveloppés d'étoffes de liber (tapa) - consistaient généralement en produits de l'activité humaine (élevage, agriculture, chasse ou pêche) ou en mèches de cheveux ${ }^{5}$. Les rites étaient exécutés par des prêtres $(k a h u)$ de Pele. Selon certains auteurs (Kamakau, 1964 : 69 ; Beckwith, 1970 : 192), il semble que ces ministres du culte de Pele se concevaient comme des descendants de la déesse. Ils mobilisaient donc vraisemblablement ce lien généalogique lorsqu'ils effectuaient des sacrifices. Toutefois, avant d'aborder cette question plus en détail, voyons quel était l'objectif de ces rites.

Ces derniers avaient deux buts. Le premier était d'éviter le déclenchement d'une éruption. Afin de mettre la déesse dans de bonnes dispositions à leur égard, les villages consacraient des offrandes à Pele au moment de la récolte des premiers fruits ou lors de festivals annuels ${ }^{6}$ (Ellis, 1979 : 250). De même, les voyageurs qui souhaitaient traverser son domaine sans encombre tentaient de se concilier la divinité en déposant des offrandes auprès de ses temples et en évitant de transgresser les tabous, comme la consommation de certaines baies (ohelo) consacrées à la déesse (Ellis, 1979 : 163 ; 250-251 ; Kalakaua, 1888 : 139). On peut proposer l'hypothèse que ces actes propitiatoires procédaient de l'échange de dons entre les dieux et les hommes. En lui offrant une partie des produits de la terre qu'elle était susceptible de détruire, ils espéraient que la déesse épargnerait celle-ci à l'avenir. Ces sacrifices étaient peut-être également destinés à favoriser la fertilité des sols volcaniques. En effet, volcanisme et fertilité étaient associés à travers la relation généalogique entre Pele et Lono-makua, son oncle, dieu de la fertilité et du renouveau

5. Les mèches de cheveux revêtaient une importance toute particulière, puisqu'elles provenaient de la tête, partie la plus sacrée d'un individu, et étaient donc considérées comme taboues ( $k a p u)$.

6. Le révérend Ellis $(1979: 250)$ précise que ses interlocuteurs lui apprirent que les habitants de Hamakua procédaient à ce genre de festival annuel, toutefois, il n'indique pas s'ils étaient les seuls, ou si d'autres districts pratiquaient aussi ce type de cérémonie collective sur une base aussi régulière. En revanche, il mentionne ailleurs l'information suivante : « the whole island was considered as bound to pay them [the volcano gods] tribute » $(1979: 173)$. Cette remarque laisse supposer que, même si tous les districts ne pratiquaient pas des cérémonies propitiatoires sur une base annuelle, tous les insulaires étaient censés payer tribut à la déesse Pele d'une manière ou d'une autre. 
complet de la nature ${ }^{7}$. L'idée est que le sacrifice des plus belles récoltes s'apparentait à l'oblation des premiers fruits telle qu'elle a été décrite par Françoise Douaire-Marsaudon à propos de Tonga (1998 : 77). Selon cet auteur, les hommes offraient aux dieux de la fertilité une toute petite partie symbolique - une pars pro toto - représentant tous les produits obtenus grâce à la fertilité de la terre, en contrepartie de tout ce qu'ils avaient reçu de ces divinités, c'est-à-dire les moyens de vivre et de perdurer. Ces hommes se plaçaient donc dans une situation de dette inextinguible vis-à-vis des dieux, ce qui était une manière de se lier à eux dans la durée 8 .

Le deuxième objectif des rites propitiatoires était de faire cesser une éruption en cours. Des offrandes étaient alors jetées dans les cratères en activité ou dans les coulées de lave menaçantes afin d'apaiser la colère divine. Dans cette perspective, ces sacrifices peuvent être interprétés comme la volonté de rétablir un lien menacé de rupture entre la déesse et les hommes. En d'autres termes, ces pratiques étaient destinées à restaurer l'harmonie entre les membres de la société, mais aussi entre ces derniers et le reste du cosmos.

\section{Les rites funéraires}

Le deuxième type de rituels liés au volcanisme prenait la forme de rites funéraires. Ceux-ci étaient effectués par un groupe de personnes qui se concevaient comme les descendants de Pele. Les textes consultés ne précisent pas leur identité ou leur rang au sein de la société hawaiienne. Toutefois, on peut retenir des sources écrites - et notamment de la description détaillée de Samuel Kamakau (1964 : 64-69) -, que les membres de ce groupe social offraient au cratère du Kilauea le corps de leurs proches parents décédés dans l'intention de transformer ces morts en aumakua pele, c'est-à-dire en " esprits du feu ancêtres ». Les esprits du feu, ou pele, étaient les êtres spirituels qui servaient la déesse Pele, attisant ses feux et les répandant sur le monde. Les aumakaua étaient des divinités tutélaires rattachées à des familles particulières, souvent en tant qu'ancêtres déifiés, mais pas toujours. Leur mécontentement était à l'origine des maladies ou de la malchance et il fallait, pour les rendre conciliants, leur offrir prières et dons (Alexander, 1891 : 39). Les rites funéraires pratiqués par certains Hawaiiens avaient donc pour but de transformer rituellement des individus qui leur étaient apparentés en esprits du feu censés conserver avec eux, au-delà de la mort, cette relation de parenté, et donc supposés respecter les obligations d'assistance qui en découlent. En effet, à Hawaii, comme dans toutes les sociétés polynésiennes, le culte des morts était primordial et les trépassés étaient conçus comme des êtres spirituels qui régulaient sans cesse la vie des vivants.

L'une des intentions des Hawaiiens, lorsqu'ils pratiquaient ces rites funéraires, était de permettre au mort de rejoindre les divinités ancestrales fondatrices de la lignée, soient la déesse Pele et ses proches. Une autre était de produire des esprits du feu susceptibles d'épargner leurs parents vivants lors d'éruptions volcaniques ; à condition, bien entendu, que ces derniers continuent à entretenir de bonnes relations avec leurs « esprits du feu ancêtres" en procédant à des sacrifices et à des prières et en respectant les règles de la vie en communauté. Ces rites funéraires, tout comme les sacrifices propitiatoires, étaient donc effectués dans la perspective d'éviter les éruptions ou d'en être épargnés.

Dans son étude sur les réactions des KuboKonai et des Bedamuni face à la sécheresse due à El-Niño, Peter Dwyer a montré que « they arose within the context of prevailing social forms and of understandings people held with respect to relationships with both environment and other people » (Dwyer et al., 2000: 521). Dans la société hawaiienne de l'époque du contact européen, les relations entre les hommes étaient basées sur le modèle de la parenté. D'une part, le lien généalogique organisait les rapports entre les individus (c'est-à-dire au sein d'un groupe de parenté comme entre les différents groupes sociaux). D'autre part, ce lien structurait les relations entre les vivants et les morts, et avec les divinités et tous les composants de l'environnement, y compris les phénomènes volcaniques. En incluant les phénomènes telluriques dans cette vaste généalogie cosmogonique englobant tous les éléments constitutifs du monde, les Hawaiiens se sont donc donnés les moyens de connaître et de gérer les phénomènes volcaniques sur des bases familières et quotidiennes. En

7. Il était la divinité principale du festival du Nouvel-An, le Makahaki, décrit entre autres par Valerio Valeri (1985) et Marshall Sahlins (1985).

8. Pour cet auteur, l'analyse qu'Alain Babadzan a développé à propos de la société tahitienne, où « l'échange entre les dieux et les hommes est placé sous le signe du do ut des : je donne pour que tu me donnes, je donne pour t'obliger à me rendre » (Babadzan, 1993 : 311), ne correspond pas à la logique polynésienne, du moins en Polynésie occidentale (Douaire-Marsaudon, 1998 : 77 note 19). Dans le cas analysé ici, il semble également difficile de croire que les Hawaiiens aient pu imaginer qu'ils pouvaient contraindre des êtres spirituels supérieurs à se soumettre ainsi à un échange égalitaire avec les hommes. 
dotant les activités volcaniques d'un principe spirituel propre et en en faisant l'un des termes d'une relation généalogique, ils ont pu instaurer avec elles une communication, un échange (Descola, 1999: 49). Cette relation d'échange permettait aux Hawaiiens d'attendre une certaine réciprocité de la part des divinités chtoniennes et donc une protection contre les phénomènes telluriques. Vulnérables face aux éruptions volcaniques, ils se sont ainsi attribués une certaine emprise sur elles.

Toutefois, ce contrôle était limité, les rites propitiatoires contenant une part d'échec intrinsèque. Par exemple, les Hawaiiens pratiquaient parfois des rites funéraires qui visaient à transformer le corps d'un chef en une "multitude» d'esprits du feu. Les parents de ce chef divisaient sa dépouille en plusieurs morceaux qu'ils distribuaient entre les districts d'Hawaii, afin qu'ils en portent chacun un au cratère, dans le but d'en faire un esprit du feu. Or, cette pratique était reconnue comme potentiellement dangereuse, puisque ces nombreux esprits du feu pouvaient parfois surgir et dévaster les terres. C'est d'ailleurs la raison pour laquelle les prêtres de Pele n'aimaient pas exécuter ce type de rites et que ceux qui cédaient aux supplications de la famille d'un chef décédé étaient appelés " destructeurs de terres » (ho'ino 'aina) ou «fauteurs de trouble du royaume" (ho'opilikia $i$ ke aupuni) ; ils étaient parfois mis à mort (Kamakau, 1964 : 65). Ces rites funéraires pouvaient avoir parfois l'effet inverse à celui escompté par les parents du mort. Ce potentiel d'échec, que les Hawaiiens attribuaient aux rituels humains, traduit sans doute la conscience de l'influence limitée de ces derniers sur les activités des puissances surnaturelles chtoniennes.

\section{Les représentations tongiennes des phénomènes volcaniques}

La communauté concernée réside actuellement à Niuafo'ou, l'île la plus septentrionale de l'archipel tongien (Fig. 2). Celle-ci a la particularité d'être la seule île volcanique active du Royaume de Tonga à être peuplée de manière permanente. Durant les deux derniers siècles, plus d'une dizaine d'éruptions ont répandu des kilomètres carrés de lave sur une portion non négligeable des terres arables de l'île. Trois ont partiellement ou entièrement détruit des villages et l'une d'entre elles a causé la mort de plusieurs villageois (en 1853). La dernière, en 1946, a eu pour conséquence l'anéantissement de la plupart des bâtiments administratifs ainsi que de la demeure de l'un des chefs nobles de l'île, poussant le gouvernement tongien à évacuer la totalité des insulaires. Ces derniers ont été réimplantés par les autorités dans l'île principale de Tongatapu, puis dans l'île méridionale de 'Eua, où des terres ont été mises à leur disposition. Douze ans après l'éruption, en 1958, ayant obtenu l'autorisation de regagner Niuafo'ou, des dizaines de familles sont retournées y vivre (Rogers, 1981, 1986). À l'heure actuelle, sept cent cinquante personnes résident dans cette île. L'éruption s'étant déroulée en 1946, il reste encore aujourd'hui des personnes âgées qui en ont été les témoins et qui en ont transmis le souvenir aux générations suivantes.

L'analyse des mémoires recueillies auprès de ces individus ${ }^{9}$ et surtout des modalités de leur transformation et de leur transmission aux jeunes générations a mis en évidence un fait significatif. Les anciens ont bien transmis un certain nombre de détails événementiels, mais ils ont surtout insisté sur les causes des éruptions, en construisant des récits explicatifs autour des faits, comme s'ils avaient voulu transmettre à leurs descendants autre chose que le seul souvenir de ces événements. Voyons donc ce qu'ils ont voulu enseigner à travers l'analyse des récits des trois éruptions qui ont détruit tout ou partie d'un village.

\section{L'éruption de 1946 à Angaha}

Pour la majorité des interlocuteurs, tous roturiers ${ }^{10}$, l'éruption a été déclenchée par Dieu $^{11}$ en réponse à des actes abusifs commis envers les gens du commun par des personnes détenant une autorité politique et sociale, c'est-à-dire les agents du gouvernement et le chef noble. Selon eux, par exemple, ce dernier avait des exigences démesurées, puisqu'il réclamait sans contrepartie porcs, ignames, nattes, etc. Si les familles ne s'exécutaient pas, le noble les chassait de leurs terres en représailles. Or, ce comportement était contraire à son rôle de chef. En effet, à Tonga, comme partout ailleurs en Polynésie, le chef a pour fonction de donner à ses sujets les moyens de vivre et de perdurer. Il doit donc savoir redis-

9. Ces témoignages ont été recueillis auprès des résidents actuels de l'île de Niuafo'ou et de personnes âgées résidant dans l'île de 'Eua, où vit toujours un grand nombre de familles originaires de Niuafo'ou.

10. Je n'ai pas eu l'occasion de recueillir l'opinion des descendants des chefs nobles ou des agents du gouvernement qui sont accusés par les actuels habitants de l'île d'avoir provoqué l'ire divine.

11. Les Tongiens ont été christianisés au milieu du XIX ${ }^{\mathrm{e}}$ siècle et se réclament aujourd'hui des valeurs chrétiennes. 


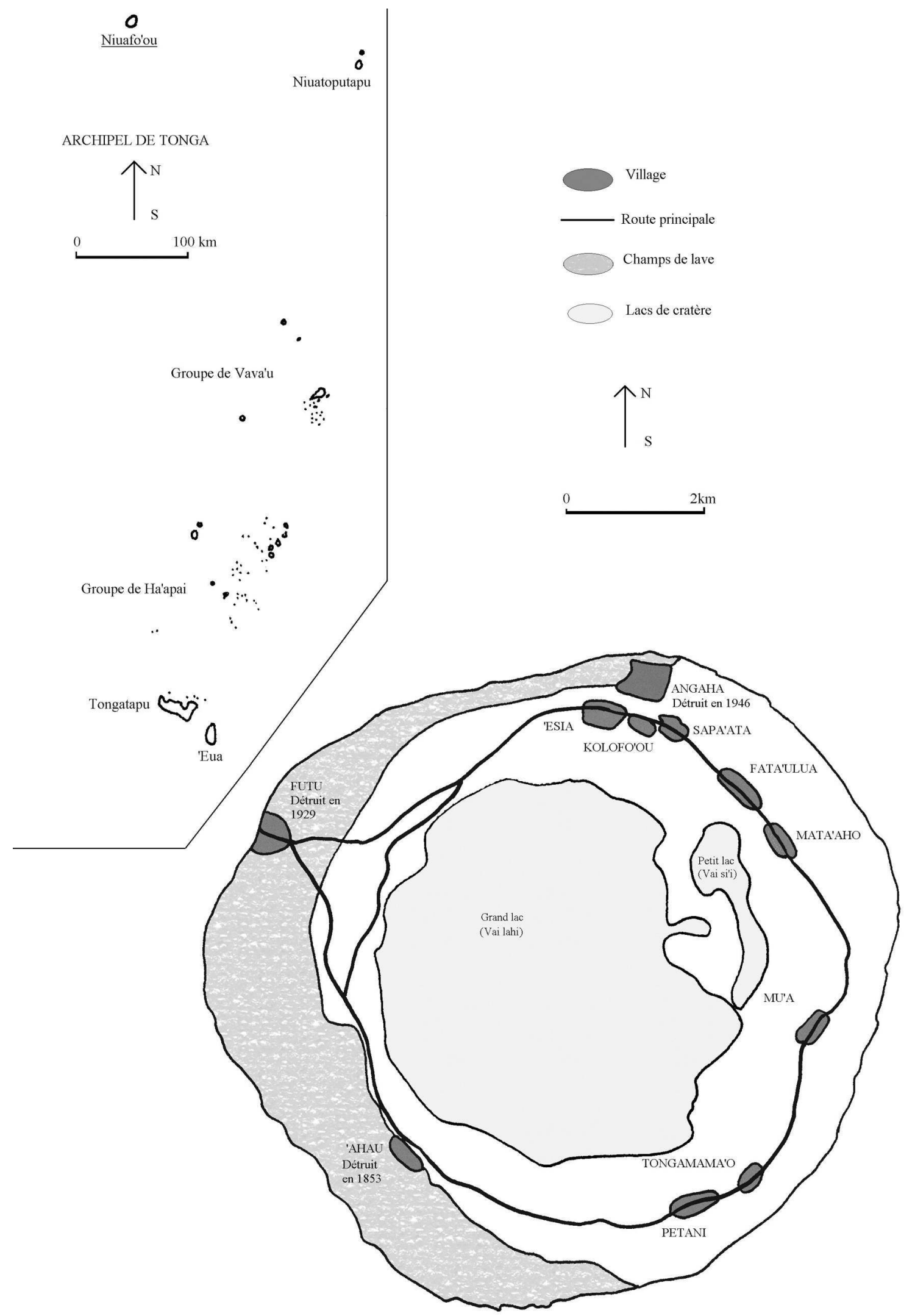

FIG. 2. - Localisation de l'île de Niuafo'ou dans l'archipel de Tonga. 
tribuer ses terres afin que leurs tenanciers en récoltent l'usufruit ${ }^{12}$. En expulsant les gens de son domaine, ce chef noble abusait donc de ses prérogatives et mettait en danger l'ordre social en privant le peuple de ses moyens de subsistance et donc de reproduction. Toujours selon les interlocuteurs, les employés du gouvernement ont quant à eux exploité le travail des habitants de l'île. Ils leur demandaient par exemple de produire de grandes quantités de coprah, avant de n'en acheter qu'une portion minimale correspondant au montant des dettes et des taxes que les gens devaient verser au gouvernement. Le reste du coprah devait être abandonné ou brûlé et les familles ne recevaient aucune compensation financière. En outre, les hommes endettés étaient souvent emprisonnés et devaient vendre leurs biens (chevaux, cochons) aux enchères bien en dessous de leur valeur - afin de payer leur libération. Or, ces pratiques sont aussi conçues par les Niuans comme une transgression du rôle normalement dévolu aux agents du gouvernement, lequel consiste à s'assurer que les gens du commun disposent de moyens de subsistance suffisants. Pour eux, l'éruption a donc été provoquée par Dieu afin de signaler et de sanctionner le non-respect de leurs rôles sociaux par les individus détenant une autorité, comportement qui représentait une mise en danger de l'ordre social et de la pérennité de la communauté niuane.

\section{L'éruption de 1929 à Futu}

En 1929, l'éruption qui a partiellement détruit le village de Futu a entraîné son abandon et la construction d'un nouveau village pour ses habitants, Kolofo'ou, littéralement, le « village nouveau ». Selon les interlocuteurs - en majorité protestants -, c'est la partie catholique du village qui a été détruite. Pour eux, Dieu a déclenché cette éruption en réponse à des péchés perpétrés par les catholiques du village ${ }^{13}$. Ces derniers sont en effet réputés n'avoir pas respecté le repos institutionnalisé du dimanche, en allant à la pêche au lieu d'assister aux services religieux. Pour les interlocuteurs, la preuve de leur culpabilité réside dans le fait que, au sein du cimetière catholique englouti par la lave, une seule tombe a été épargnée, celle d'un individu protestant enterré là par sa famille catholique. À leurs yeux, sa tombe n'a pas été détruite parce qu'il était protestant et donc innocent des activités répréhensibles attribuées aux catholiques.

\section{L'éruption de 1853 à 'Ahau}

En 1853, l'éruption qui a enseveli le village de 'Ahau a décimé une bonne partie de sa population. Si les estimations du nombre de victimes varient selon les sources écrites ${ }^{14}$, les informateurs affirment que tous les habitants ont été tués, à l'exception de trois ou quatre individus. Cette éruption est aujourd'hui interprétée comme une punition divine à l'encontre d'habitants aux mœurs immorales. Ces gens sont réputés s'être adonnés à des actes sexuels illicites comme l'adultère au sein de l'église, lorsqu'ils s'y réfugiaient pour échapper aux moustiques. Dans ce cas, la preuve donnée par les Niuans est que les seuls individus censés ne pas s'être livrés à ces activités réprouvées ont survécu à l'éruption. Les jeunes générations justifient aussi ce point de vue en ajoutant que la lave a jailli au cœur de l'église où dormaient les villageois coupables.

\section{Interprétations et représentations des éruptions volcaniques}

Ces interprétations des éruptions sont celles qui sont les plus couramment données aujourd'hui par une majorité importante des individus originaires de Niuafo'ou ${ }^{15}$. Il est difficile de dire exactement quelles étaient les représentations des éruptions volcaniques avant le contact avec les Européens. Cependant, on sait que les perturbations qui touchaient des groupes d'insulaires comme les épidémies, les cyclones, les famines ou les guerres, étaient attribuées à des

12. Cette obligation traditionnelle qui est faite aux nobles de donner leurs terres en usufruit à leurs sujets a été légalisée par les lois constitutionnelles mises en place par le roi George Tupou I dans la deuxième moitié du XIX ${ }^{\mathrm{e}}$ siècle (Latukefu, 1975).

13. Certains interlocuteurs catholiques ont réfuté ce type d'explication en affirmant que la moitié catholique du village a été détruite parce qu'elle se situait au bord de la plage, tandis que la partie protestante était située sur une colline et se trouvait donc hors de portée des coulées de lave.

14. Une vieille femme confia, en 1930, au volcanologue Thomas Jaggar, que les deux tiers de la population, soit soixante à soixante-dix individus, avaient péri (Jaggar, 1935 : 96). Un commerçant anglais installé à Niuafo'ou à la même époque affirme que seulement huit personnes n'en réchappèrent pas (Ramsay, $1938: 259$ ). Un article publié dans le Journal officiel tongien, à l'occasion du centenaire de l'éruption, donne quant à lui le chiffre moyen de vingt-cinq morts (Tonga Chronicle, 9 septembre $1953: 10)$.

15. Parce qu'ils partagent également aujourd'hui en partie un bon nombre de connaissances d'origine scientifique occidentale qui leur ont été transmises à l'école, les habitants de Niuafo'ou estiment aussi que certaines éruptions passées se sont déclenchées indépendamment du contrôle direct de Dieu, de manière naturelle (les Tongiens disent fakanatula, translittération du mot anglais naturally). Toutefois, cet aspect plus sécularisé des représentations des éruptions et de l'environnement qui coexiste avec les conceptions décrites dans cet article ne sera pas abordé ici, car il en déborde le cadre. 
divinités originelles et à des ancêtres divinisés. Ces entités spirituelles déclenchaient ces catastrophes afin de châtier le non-respect, par certains groupes sociaux, de leurs obligations sociales, comme par exemple l'oubli de certaines cérémonies propitiatoires dédiées à un ancêtre déifié par les membres d'un groupe de parenté. Lorsqu'un seul individu était à l'origine d'une transgression, il était puni par la maladie ou la mort - de lui-même ou de l'un de ses proches (Martin, 1991 : 204-213). Or, après la christianisation (au milieu du XIX ${ }^{\mathrm{e}}$ siècle), bon nombre de ces sanctions surnaturelles, y compris les éruptions volcaniques, ont été mises au compte de Dieu.

Ainsi, pour les habitants de Niuafo'ou, Dieu provoque les éruptions afin de signaler et de punir les transgressions de l'ordre social, en tant que créateur soucieux de réguler la vie de ses créatures, les hommes. Dans cette logique, les insulaires affirment que maintenir l'harmonie sociale en respectant les règles de la vie en société ${ }^{16}$ permet de se concilier Dieu et donc d'éviter, dans une certaine mesure, le déclenchement d'éruptions destructrices. Les normes sociales correspondent à un ensemble de valeurs " which are seen as traditional values that have been strengthened by Christianity [...]. They are explicitly defined by many Tongans as intrinsic to their cultural identity » (Morton, 1996: 80). Selon l'auteur, ces principes moraux incluent notamment l'amour ('ofa), le respect (faka'apa'apa) et l'obéissance (talangofua). Parce que ces valeurs traditionnelles correspondent aux préceptes chrétiens, on retrouve généralement dans les discours des interlocuteurs l'idée selon laquelle respecter les règles de la vie en société signifie obéir aux enseignements bibliques en se gardant de pécher. Les Niuans estiment également qu'il faut prier régulièrement Dieu afin de l'honorer, de lui demander d'épargner souffrances et épreuves aux hommes, et de leur accorder grâces et bienfaits. Une semaine spéciale de prières (uike lotu) est même observée chaque année en juin par les membres des églises protestantes. Elle correspond à la date d'un tremblement de terre meurtrier qui, en juin 1977, frappa durement Tonga. Plusieurs services sont organisés tous les jours de cette semaine afin de rendre hommage aux victimes du séisme et de supplier Dieu de les épargner à l'avenir, en faisant cesser ce type de catastrophe. Les prières individuelles et collectives peuvent donc être analysées comme des actes propitiatoires, dont l'objectif est de mettre la divinité dans de bonnes dispositions à l'égard des hommes et donc de se protéger aussi contre les éruptions et les séismes.

Toutefois, les interlocuteurs reconnaissent également que la prière ou les efforts individuels opérés par chacun afin de préserver l'harmonie sociale ne garantissent pas forcément la maîtrise des futures activités éruptives de l'île. Deux habitants de Niuafo'ou ont exprimé ce sentiment d'incertitude en ces termes :

« Si quelque chose de mal arrive en dépit des prières, c'est que Dieu veut mettre notre foi à l'épreuve. »

« Parce qu'il veut nous montrer sa puissance. »

Ces gens admettent ainsi que leurs actions ont une limite parce qu'au bout du compte, c'est à une entité surnaturelle infiniment supérieure que revient le contrôle de ce type de perturbation environnementale. Or, les hommes étant dans l'impossibilité d'appréhender l'incommensurable volonté divine, ils sont par conséquent incapables d'en influencer totalement les actes.

\section{Comparaison des approches hawaiienne et tongienne}

Dans les deux sociétés polynésiennes étudiées, Hawaii à l'époque du contact européen et Tonga (Niuafo'ou) aujourd'hui, on retrouve la conception d'une relation, d'une interaction entre les hommes, les phénomènes volcaniques et le divin. Cependant, à Hawaii durant la période du contact, ces composants de l'environnement étaient considérés et traités comme des êtres vivants et pensants, interagissant entre eux et avec les hommes. À Niuafo'ou à l'heure actuelle, il n'existe pas une telle personnalisation des éléments naturels. On n'y retrouve qu'une seule figure du sacré, le dieu chrétien. En revanche, comme à Hawaii, les éruptions volcaniques signalent la colère divine. Toutefois, à la différence de Hawaii, ces éruptions ne sont pas considérées comme les manifestations physiques de divinités agissant d'elles-mêmes. Elles sont au contraire conçues comme des moyens de communication entre les hommes et Dieu. Dieu a créé ces éléments naturels, il les utilise pour punir les transgressions de l'ordre social, il représente ses « instruments », mais il ne s'y incarne pas.

Au plan des représentations de l'éventualité de futures éruptions volcaniques, ces deux sociétés ont dans l'idée que chacun est responsable du maintien de l'ordre social; une responsabilité médiatisée par une entité surnaturelle bien plus 
puissante que les hommes et capable de provoquer des perturbations qui ne sont pas forcément maitrisables par ces derniers. Toutefois, tout en reconnaissant l'imprévisibilité intrinsèque des divinités et des phénomènes environnementaux qu'elles peuvent contrôler, ces hommes ont imaginé des rapports sociaux qui les unissent à ces déités et leur permettent de communiquer avec elles. Par l'intermédiaire de ces relations, ils ont alors pu concevoir des pratiques rituelles propitiatoires destinées à se concilier ces divinités afin qu'elles évitent de déclencher des éruptions, ou qu'elles fassent cesser celles qu'elles ont provoquées. On peut alors sans doute interpréter l'idée d'un potentiel d'échec entourant ces pratiques humaines comme la reconnaissance implicite, au plus profond des actes religieux, du caractère limité de leurs propres actions sur ce type de phénomènes et sur les divinités qui en sont à l'origine. C'est ce " principe de réalité » qui fait que les relations que ces insulaires entretiennent avec les composants géophysiques de leur environnement sont teintées d'incertitude et nécessitent une vigilance constante.

\section{Conclusion}

Malgré leur éloignement et les différents contextes qui les caractérisent, les deux sociétés polynésiennes concernées ont pour point commun de traiter les phénomènes volcaniques comme des moyens de communication entre les puissances surnaturelles qui les contrôlent et les hommes. À Hawaii comme à Tonga, les éruptions sanctionnent les transgressions des règles de la vie en communauté et servent à rappeler aux hommes qu'il est de leur devoir de préserver l'ordre socio-cosmique. À travers ces conceptions similaires, ces deux sociétés polynésiennes, loin de traiter les volcans et leurs activités associées comme des phénomènes hors de leur portée, les ont donc transformés en acteurs de leur vie sociale, les insérant ainsi dans leur champ relationnel.

\section{BIBLIOGRAPHIE}

AleXANDER William de Witt, 1891. A brief history of the Hawaiian people, New York, American book Cie.

Babadzan Alain, 1993. Les dépouilles des dieux. Essai sur la religion tahitienne à l'époque de la découverte, Paris, Éditions de la maison des sciences de l'homme.
BECKwICK Martha, 1970. Hawaiian Mythology, Honolulu, University of Hawaiian Press.

Descola Philippe, 1999. Les natures sont dans la culture, Sciences humaines hors série 23, pp. 46-49.

DouaIre-Marsaudon Françoise, 1998. Les premiers fruits. Parenté, identité sexuelle et pouvoirs en Polynésie occidentale (Tonga, Wallis et Futuna), Paris, Éditions de la maison des sciences de l'homme.

Dunis Serge, 1990. Ethnologie d'Hawaii. " Homme de la petite eau, femme de la grande eau », Paris, L'Harmattan.

Dwyer Peter and Monica Minnegal, 2000. Responses to a drought in the interior Lowlands of Papua New Guinea: A comparison of Bedamuni and Kubo-Konai, Human Ecology 28, 4, pp. 493-526.

ELLIS William (révérend), 1979. Journal of William Ellis, Ruthland, Vermont \& Tokyo, C. E. Tuttle Cie.

Handy Craighill E.S., 1927. Polynesian religion, Honolulu, Bernice P. Bishop Museum, Bulletin 34.

JAGGAR Thomas A., 1935. Living on a volcano. An unspoiled patch of Polynesia is Niuafoo, Nicknamed "Tin Can Island" by stamp collectors, The National Geographic Magazine 48, pp. 91-106.

KalaKaua David (His Hawaiian Majesty), 1888. The legends and myths of Hawaii, New York, Charles E. Webster \& Cie.

Kamakau Samuel M., 1964. Ka fo'e ka kahiko. The people of old, Honolulu, Bishop Museum Press.

KamaKau Samuel M., 1976. The works of the people of old, Honolulu, Bishop Museum Press.

LATUKEFU Sione, 1975. The tongan Constitution: a brief history to celebrate its centenary, Nuku'alofa, Tonga Tradition Committee Publication.

Linge Gabriel, 1975. L'étrange destin de Hawaii, Paris, Robert Laffont.

Martin John, 1991. Tonga Islands. William Mariner's account, Nuku'alofa, Vava'u Press.

Morton Helen, 1996. Becoming Tongan. An ethnography of childhood, Honolulu, University of Hawaiian Press.

Pukui Mary Kawena and Alfons L. KoRn, 1993. The echo of our song. Chants and poems of the Hawaiians, Honolulu, University of Hawaiian Press.

Quesada Cécile, 2000. Des Polynésiens face aux volcans et séismes. L'appropriation sociale des phénomènes telluriques à Tonga, Hawaii et en NouvelleZélande au moment du contact européen, mémoire de DEA, EHESS-Marseille.

Ramsay Charles, 1938. Tin Can Island. A story of Tonga and the swimming mail man of the south seas, Plymouth, Hurst \& Blackett.

Rogers Garth, 1981. The evacuation of Niuafo'ou, an Outlier in the Kingdom of Tonga, Journal of Polynesian History 16, 3, pp. 149-163.

RoGERs Garth, 1986. The fire has jumped. Eyewitness accounts of the eruption and evacuation of 
Niuafo'ou, Tonga, Suva, University of the South Pacific, Institute of Pacific Studies.

SALHINs Marshall, 1985. Islands of History, Chicago and London, University of Chicago Press.

SALHINS Marshall, 1995. How natives think, Chicago and London, University of Chicago Press.

Tonga Chronicle, 1953 (9 septembre).
VALERI Valerio, 1985. Kinship and Sacrifice. Ritual and Society in Ancient Hawaii, Chicago and London, University of Chicago Press.

Westervelt William D., 1963. Hawaiian legends of Volcanoes, Ruthland, Vermont and Tokyo, C.E. Tuttle. 
